\title{
Doping of a Spin-1 Chain: An Integrable Model
}

\author{
Holger Frahm, ${ }^{1}$ Markus P. Pfannmüller, ${ }^{1}$ and A. M. Tsvelik ${ }^{2}$ \\ ${ }^{1}$ Institut für Theoretische Physik, Universität Hannover, D-30167 Hannover, Germany \\ ${ }^{2}$ Department of Physics, University of Oxford, 1 Keble Road, Oxford, OX1 3NP, United Kingdom
}

(Received 12 March 1998)

An exactly soluble model describing a spin $S=1$ antiferromagnetic chain doped with mobile $S=1 / 2$ carriers is constructed. In its continuum limit the undoped state is described by three gapless Majorana fermions composing the SU(2) triplet. Doping adds a scalar charge field and a singlet Majorana fermion with different velocity. We argue that this mode survives when the Haldane gap is added. [S0031-9007(98)07027-6]

PACS numbers: 71.10.Pm, 75.10.Jm, 75.50.Ee

It is well known that mobile holes introduced into antiferromagnetic Mott insulators by means of doping cause frustration which seriously affects the magnetic properties of the system. In this paper we study a doped spin-1 chain which in the presence of mobile holes interpolates between $S=1$ and $S=1 / 2$ states. According to Refs. [1-3] this situation is realized in the carrier doped Haldane system $\mathrm{Y}_{2-x} \mathrm{Ca}_{x} \mathrm{BaNiO}_{5}$ [4]: For $x=0$ the interactions between the spin- $1 \mathrm{Ni}^{2+}$ ions are well described by a Heisenberg model, upon doping mixing of the $S=1 / 2$ holes on the Oxygen sites leads to a low energy doublet state in an effective one-band Hamiltonian which can move in the $S=1$ background.

The model we study is an integrable model whose Hamiltonian closely resembles the Hamiltonian introduced in [2]:

$$
\begin{gathered}
\mathcal{H}=\sum_{n=1}^{L}\left\{\mathcal{H}_{n, n+1}^{\mathrm{exch}}+\mathcal{H}_{n, n+1}^{\mathrm{hopp}}\right\} \\
\mathcal{H}_{i j}^{\text {exch }}=\frac{1}{2}\left(\frac{1}{S_{i} S_{j}} \mathbf{S}_{i} \cdot \mathbf{S}_{j}-1\right. \\
\left.\quad+\delta_{S_{i} S_{j}, 1}\left[1-\left(\mathbf{S}_{i} \cdot \mathbf{S}_{j}\right)^{2}\right]\right), \\
\mathcal{H}_{i j}^{\text {hopp }}=-\left(1-\delta_{S_{i}, S_{j}}\right) \mathcal{P}_{i j}\left(\mathbf{S}_{i} \cdot \mathbf{S}_{j}\right) .
\end{gathered}
$$

Here $\mathbf{S}_{i}^{2}=S_{i}\left(S_{i}+1\right)$ with $S_{i}=1$ or $1 / 2$ and $\mathcal{P}_{i j}$ permutes the spins on sites $i$ and $j$. Comparing (1) to the effective one-band Hamiltonian for the doped $\mathrm{Ni}$ oxide given in [2],

$$
\mathcal{H}_{i j}=\delta_{S_{i} S_{j}, 1} J \mathbf{S}_{i} \cdot \mathbf{S}_{j+1}-\mathcal{P}_{i j}\left(\mathbf{S}_{i} \cdot \mathbf{S}_{j}+\frac{1}{2}\right),
$$

we find several differences. First, the spin exchange between $S=1$ sites contains biquadratic terms giving the spin-1 Takhtajan-Babujian chain for hole concentration $x=0$. Therefore in the undoped limit the spectrum is gapless and one may think that the most spectacular feature of the doped $S=1$ chain-filling the gap with holes, is lost in our model. We shall see later, however, that it is possible to reintroduce a gap in the continuous limit where we have a field theoretical description of the model. Second, due to a hidden supersymmetry of the model (see below) the ratio between the exchange integral and the hopping is fixed in (1). We do not expect this to be a serious restriction on physical properties of the model. Third, hopping processes such as $\left|1_{i} \downarrow_{j}\right\rangle \rightarrow\left|\downarrow_{i} 1_{j}\right\rangle$ are allowed. Finally, the integrable model contains an antiferromagnetic exchange interaction between nearest neighbor hole states which is responsible for the absence of a ferromagnetic phase of (2) for sufficiently large hole concentration. Such an interaction has, however, been considered in Ref. [3] to improve agreement with the experiments at $x=1$.

In the following we will use the exact solution of (1) to deduce an effective field theory for the low-energy sector of the system which will enable us to study possible relevant perturbations. The low-energy limit of the undoped system $(x=0)$ is well known to be a realization of an SU(2) level $k=2$ Wess-Zumino-Novikov-Witten (WZNW) model with central charge $c=3 / 2$. This model is equivalent to a model of three massless Majorana fermions composing an $\mathrm{SU}(2)$ triplet [5]. A completely filled $(x=1)$ band corresponds to the $S=1 / 2$ chain which in the low-energy limit is equivalent to the $\mathrm{SU}(2)_{1} \mathrm{WZNW}$ model. For finite doping we find, as may be expected, one free bosonic mode in the charge sector. The spin sector, however, turns out to be rather unusual containing a direct sum of $c=3 / 2$ and $c=1 / 2$ models with different velocities. Thus doping generates the fourth Majorana fermion - a feature observed in various models related to two-channel Kondo physics [6,7].

The model (1) is constructed from solutions to the Yang Baxter (YBE) equation invariant under the action of the graded Lie algebra $g l(2 \mid 1)$ in the "atypical" representation $[S]_{+}[8]$. These representations contain two multiplets of spin $S$ and $(S-1 / 2)$ with respect to the $\mathrm{SU}(2)$-subalgebra of $g l(2 \mid 1)$. A well known example for a lattice model obtained from the $[1 / 2]_{+}$-representation in this approach is the supersymmetric $t-J$ model (see, e.g., [9]). Denoting the generators of the $\mathrm{SU}(2)$ subalgebra by $S^{a}$, the U(1)-charge operator by $B$ and the remaining fermionic generators of $g l(2 \mid 1)$ by $V^{ \pm}, W^{ \pm}$(which create and annihilate the holes, see [8]) the local $\mathcal{L}$ operator of the quantum inverse scattering method reads 


$$
\mathcal{L}(\mu) \propto\left(\begin{array}{ccc}
\mu+2 i B & i \sqrt{2} W^{-} & i \sqrt{2} W^{+} \\
i \sqrt{2} V^{+} & \mu+i\left(B+S^{z}\right) & -i S^{+} \\
-i \sqrt{2} V^{-} & -i S^{-} & \mu+i\left(B-S^{z}\right)
\end{array}\right) .
$$

The spectrum of the transfer matrix for a vertex model with $L$ of these weights per row is obtained by means of the algebraic Bethe ansatz [10]. Starting from the fully polarized state of spin- $S$ multiplets on each site we consider states with $N_{h}$ holes (generating sites with spin $S-1 / 2$ ) and magnetization $M^{z}=L S-\frac{1}{2} N_{h}-$ $N_{\downarrow}$. This leads to an auxiliary eigenvalue problem for an inhomogenous graded six-vertex model on a lattice of $n=N_{h}+N_{\downarrow}$ sites which is solved by means of a second Bethe Ansatz through addition of states with fermionic grading (holes) to its eigenstate with $n=N_{\downarrow}$ (for details see, e.g., [9]). As a result the spectrum of the supersymmetric vertex model is parametrized by the roots of the following set of Bethe-ansatz equations (BAE)

$$
\begin{aligned}
\left(\frac{\lambda_{j}+i S}{\lambda_{j}-i S}\right)^{L} & =\prod_{k \neq j}^{N_{h}+N_{\downarrow}} \frac{\lambda_{j}-\lambda_{k}+i}{\lambda_{j}-\lambda_{k}-i} \prod_{\alpha=1}^{N_{h}} \frac{\lambda_{j}-\nu_{\alpha}-\frac{i}{2}}{\lambda_{j}-\nu_{\alpha}+\frac{i}{2}}, \\
j & =1, \ldots, N_{h}+N_{\downarrow} \\
1 & =\prod_{k=1}^{N_{h}+N_{\downarrow}} \frac{\nu_{\alpha}-\lambda_{k}+\frac{i}{2}}{\nu_{\alpha}-\lambda_{k}-\frac{i}{2}}, \\
\alpha & =1, \ldots, N_{h} .
\end{aligned}
$$

For $S=1 / 2$, where the model becomes the supersymmetric $t$ - $J$ model, and Eqs. (4) are Sutherland's form of the BAE [11]. The grading of the underlying algebra allows to construct two more sets of (equivalent) BAE $[9,12]$. Equations (4) are most convenient for our analysis, however.

To construct a local Hamiltonian for $S=1$ we project the graded tensor product $\mathcal{L}_{a}\left(\mu+\frac{i}{2}\right) \hat{\otimes} \mathcal{L}_{b}\left(\mu-\frac{i}{2}\right)$ in two copies $V_{a}, V_{b}$ of the matrix space of (3) onto the $[1]_{+}$representation in $V_{a} \otimes V_{b}$. The result $\mathcal{L}_{[1]_{+}}(\mu)$ of this fusion $[13,14]$ defines a new vertex model with eigenstates again parametrized by the roots of (4). The logarithmic derivative of its transfer matrix at $\mu=0$ is the Hamiltonian (1) with eigenvalues

$$
\begin{aligned}
E\left(\left\{\lambda_{j}\right\},\left\{\nu_{\alpha}\right\}\right)-H M^{z}-\tilde{\mu} N_{h}= & \sum_{k=1}^{N_{h}+N_{\downarrow}}\left(H-\frac{2}{\lambda_{k}^{2}+1}\right) \\
& -\sum_{\alpha=1}^{N_{h}}\left(\mu+\frac{1}{2} H\right) \\
& -L H
\end{aligned}
$$

[we have added an external magnetic $H$ field and a (hole) chemical potential $\tilde{\mu}=\mu+1$ to the Hamiltonian].

In the thermodynamic limit the solutions of the BAE (4) are real hole rapidities $\nu$, whose density we denote as $\rho(\nu)$ and complex $\lambda$ strings with densities $\sigma_{n}(\lambda)$ $(n=1,2, \ldots)$. The equations for the densities are

$$
\begin{aligned}
\delta_{n, 2} s(x) & =\sigma_{n}(x)+C_{n m} * \tilde{\sigma}_{m}(x)-\delta_{n, 1} s * \rho(x), \\
a_{2} s(x) & =\tilde{\rho}(x)+\left[1+a_{2}\right]^{-1} * \rho(x)+s * \tilde{\sigma}_{1}(x),
\end{aligned}
$$

where all densities marked by tilde correspond to holes, $f * g(x)$ denotes a convolution, $s(x)=1 /(2 \cosh \pi x)$, $a_{n}(x)=(2 n / \pi) /\left(4 x^{2}+n^{2}\right)$ and

$$
C_{n m}(x)=\delta_{n m} \delta(x)-\left(\delta_{n+1, m}+\delta_{n-1, m}\right) s(x) .
$$

Writing the energy (5) in terms of densities of $\lambda$ holes and $\rho$ we obtain

$$
\begin{aligned}
E / L= & -2-\int d x\left[2 \pi\left(a_{2} * s\right)+\mu\right] \rho(x) \\
& +\int d x 2 \pi s(x) \tilde{\sigma}_{2}(x)-\lim _{n \rightarrow \infty} H n \int d x \tilde{\sigma}_{n}(x) .
\end{aligned}
$$

By minimization of the free energy we obtain the thermodynamic Bethe ansatz (TBA) equations for the energies $\epsilon_{n}=T \ln \left(\tilde{\sigma}_{n} / \sigma_{n}\right)$ of $\lambda$ strings and $\kappa=T \ln (\tilde{\rho} / \rho)$ for the hole rapidities

$$
\begin{aligned}
\epsilon_{n}(x)= & T s * \ln \left[1+e^{\epsilon_{n-1}(x) / T}\right]\left[1+e^{\epsilon_{n+1}(x) / T}\right] \\
& -2 \pi \delta_{n, 2} s(x)-\delta_{n, 1} T s * \ln \left[1+e^{-\kappa(x) / T}\right],
\end{aligned}
$$

subject to the condition $\lim _{n \rightarrow \infty}\left(\epsilon_{n} / n\right)=H$ and

$$
\begin{aligned}
-\left[2 \pi a_{2} * s(x)\right. & +\mu]-T s * \ln \left[1+e^{\epsilon_{1}(x) / T}\right] \\
& =\kappa(x)+T R * \ln \left[1+e^{-\kappa(x) / T}\right],
\end{aligned}
$$

where $R=a_{2} *\left(1+a_{2}\right)^{-1}$. In terms of these functions the free energy reads

$$
\begin{aligned}
F / L= & -2-T \int d x s(x) \ln \left[1+e^{\epsilon_{2}(x) / T}\right] \\
& -T \int d x\left(a_{2} * s\right)(x) \ln \left[1+e^{-\kappa(x) / T}\right] .
\end{aligned}
$$

For temperatures $T \ll H$ the energies $\epsilon_{n>2}$ can be eliminated from (8), giving the following equation for $\epsilon_{2}$ :

$$
\begin{aligned}
\epsilon_{2}(x)-T R * \ln \left[1+e^{\epsilon_{2}(x) / T}\right]= & -2 \pi s(x)+H / 2 \\
& +T s * \ln \left[1+e^{\epsilon_{1}(x) / T}\right] .
\end{aligned}
$$

Together with the $n=1$ equation from (8) and (9) this equation determines the low-temperature phase diagram of the system (Fig. 1). For sufficiently large negative $\mu$ Eq. (9) implies $\kappa>0$ corresponding to vanishing hole density. The remaining TBA coincide with those for the integrable $S=1$ magnet [15,16]. Choosing $\mu>H / 2$ we find $\kappa<0$ and $\epsilon_{1}<0$ which implies a hole density $x=1$. The resulting TBA for $\epsilon_{n \geq 2}$ are those of the $S=1 / 2$ Heisenberg chain. Similar considerations for intermediate $\mu$ yield the complete phase diagram and lead us to identify $\kappa$ and $\epsilon_{1}$ as the energy of the charge and spin modes associated with the mobile carriers while $\epsilon_{2}$ is the magnetic mode of the background spins. 


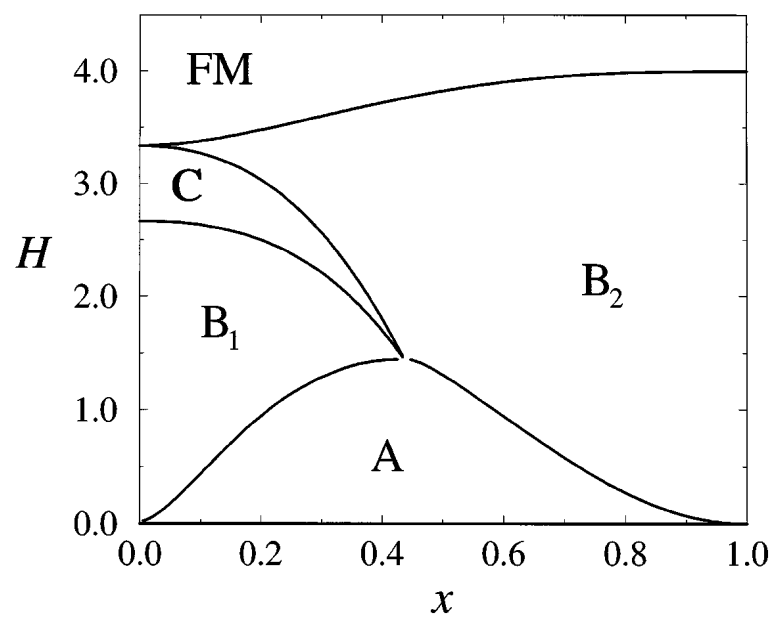

FIG. 1. Low temperature phase diagram of the doped chain in a magnetic field $H \gg T$ : the saturated ferromagnetic phase is labeled FM. At intermediate fields the low-energy properties of the system are determined by gapless bosonic modes with energies $\kappa\left(A, B_{1}\right), \epsilon_{1}\left(A, B_{2}, \mathrm{FM}\right)$, and $\epsilon_{2}\left(A, B_{1,2}, C\right)$. The $H=0$ phase is discussed in the main text.

We now concentrate on the case of finite doping in a vanishing magnetic field which corresponds to chemical potentials $\pi-4<\mu<0$. In this regime we have $\kappa<$ 0 for $|x|<Q$. For temperatures smaller than the Fermi energy of holes $T \ll-\mu$ one can replace $\kappa$ in Eq. (8) by its zero temperature value $\kappa_{0}(x)$ and the free terms by their asymptotics. As a result we get

$$
\begin{aligned}
\epsilon_{n}(x)= & T s * \ln \left[1+e^{\epsilon_{n-1}(x) / T}\right]\left[1+e^{\epsilon_{n+1}(x) / T}\right] \\
& -2 \pi \delta_{n, 2} e^{-\pi|x|}-2 \pi A \delta_{n, 1} e^{-\pi|x|}
\end{aligned}
$$

where $A=-(2 \pi)^{-1} \int_{-Q}^{Q} d y e^{\pi y} \kappa_{0}(y)$. To study the specific thermodynamic properties of the present model we have to separate the contributions to the free energy stemming from the charge sector from those due to the $\epsilon_{n}$. Considering low temperatures again the leading contributions to $\kappa$ come from the vicinity of the Fermi wave vectors $\pm Q$. In this region one can safely neglect contributions to Eq. (9) from $\epsilon_{1}$ and rewrite it as

$$
\begin{array}{r}
-\left[2 \pi a_{2} * s(x)+\mu\right]-T R * \ln \left[1+e^{-|\kappa(x)| / T}\right] \\
=\kappa(x)-\int_{-Q}^{Q} d y R(x-y) \kappa(y),
\end{array}
$$

where $Q$ is determined by the condition $\kappa( \pm Q)=0$. Using the procedure introduced by Takahashi [17], we can rewrite the free energy (10) as $F / L=E_{0} / L+f_{1}+f_{2}$ where $E_{0}$ is the ground-state energy and

$$
\begin{gathered}
f_{1}=-T \int d x \rho_{0}(x) \ln \left[1+e^{-\left|\kappa_{0}(x)\right| / T}\right] \approx-\pi T^{2} / 6 v, \\
f_{2}=-T \int d x s(x) \ln \left[1+e^{\epsilon_{2}(x) / T}\right] \\
-T \int d x\left(s * \rho_{0}\right)(x) \ln \left[1+e^{\epsilon_{1}(x) / T}\right] .
\end{gathered}
$$

Here $\rho_{0}(x)$ is the density of hole rapidities at $T=0$

$$
\rho_{0}(x)-\int_{-Q}^{Q} d y R(x-y) \rho_{0}(y)=a_{2} * s(x)
$$

and $v$ is the velocity of the charge mode $\kappa$

$$
v=\left.\frac{1}{2 \pi \rho_{0}(Q)} \frac{\partial \kappa_{0}}{\partial x}\right|_{x=Q} .
$$

In the low- $T$ limit $T \ll-\mu$ where this form is valid one may replace $s(x)$ and $s \rho_{0}(x)$ in (15) by their asymptotics. Now we have TBA equations in such form that the spin sector is manifestly decoupled from the charge one. The free energy of the latter is given by Eq. (14) representing a scalar bosonic mode, while Eqs. (12) and (15) describe the thermodynamics of the spin sector. Such TBA equations (i.e., with two driving terms) arise also in other systems combining different spins [18] and in the two-channel Kondo model with a channel anisotropy [19]. As shown in Ref. [7] such a two-channel Kondo model can be written in terms of four Majorana fermions. These considerations lead us to hypothesize that the effective low-energy theory for the Hamiltonian (1) at small doping is

$$
\begin{aligned}
\mathcal{H}_{\mathrm{eff}} & =\int d x[\mathcal{H}+\overline{\mathcal{H}}] \\
\mathcal{H} & =-\frac{i}{2} \sum_{a=1}^{3} v_{2} \chi_{a} \partial_{x} \chi_{a}-\frac{i}{2} v_{1} \chi_{0} \partial_{x} \chi_{0} \\
& +g \chi_{0} \chi_{1} \chi_{2} \chi_{3}
\end{aligned}
$$

and a similar expression $\overline{\mathcal{H}}(\{\bar{\chi}\})$ for the left movers. The fields $\chi_{0}, \chi_{a}$ are Majorana fermions and the marginal coupling $g$ between two sectors in (17) is the only one possible by symmetry (we have omitted marginally irrelevant interactions between sectors with different chirality). A magnetic field couples to the term $i \int\left(\beta \chi_{0} \chi_{a}+\epsilon_{a b c} \chi_{b} \chi_{c}\right)$ quadratic in the fermions with some constant $\beta$.

The parameters of the effective theory have to be extracted from the TBA at small doping. This corresponds to $A \ll 1$ and implies that the two terms in (15) are dominated by contributions from the region $\pi x \sim \ln (2 \pi / T)$ (where $\left|\epsilon_{2}\right| \sim T$ ) and by $\pi x \sim \ln (2 \pi A / T)$, respectively. This separation of scales allows one to obtain the small $A$ behavior of (15) at low temperatures

$$
\begin{aligned}
f_{2}= & -\frac{\pi T^{2}}{6 v_{1}}\left(\frac{1}{2}-\frac{3 A}{4 \pi} \ln A\right) \\
& -\frac{\pi T^{2}}{6 v_{2}}\left(\frac{3}{2}+\frac{3 A}{4 \pi} \ln A\right)+\ldots
\end{aligned}
$$

For $A=0$ this is the free energy of a single Majorana fermion $\left[\chi_{0}\right.$ in (17)] with velocity $v_{1}$ and that of the $\mathrm{SU}(2)_{2}$ WZNW model with velocity $\boldsymbol{v}_{2}$ which can be expressed in terms of massless triplet of Majorana fermions [5]. The ratio of the velocities is given by

$$
v_{1} / v_{2}=-\frac{1}{2 \pi} \frac{\int_{-Q}^{Q} d y e^{\pi y} \kappa_{0}(y)}{\int_{-Q}^{Q} d y e^{\pi y} \rho_{0}(y)} .
$$


The coupling constant $g$ needs to be chosen such that it produces the $A$ dependence of $f_{2}$. Similarly, the parameter $\beta$ can be determined from the $T=0$ magnetic susceptibility. At small $A \ll 1$ we get

$$
\chi=\frac{1}{\pi}\left(\frac{1}{v_{2}}-\frac{1}{v_{1}} \frac{A}{2 \pi} \ln A+\ldots\right)
$$

giving $\beta^{2}=A / 2 \pi$. In Fig. 2 some of these quantities are given as a function of the hole concentration $x$.

The simplest $\mathrm{SU}(2)$ invariant perturbation of (17) is a mass term $i \int d x\left(m \sum_{a=1}^{3} \bar{\chi}_{a} \chi_{a}+M \bar{\chi}_{0} \chi_{0}\right)$ for the particles in the spin sector (recall that the model of three massive Majorana fermions is a good continuous approximation for the $S=1$ Heisenberg antiferromagnet [20]). Choosing $M=0$ we get a situation with a Haldane gap in the triplet sector and gapless singlet excitations. This may be a good description for the magnetic states inside the Haldane gap observed in the doped Ni oxide [4].

To summarize, we have introduced an integrable model describing a magnetic system which interpolates between
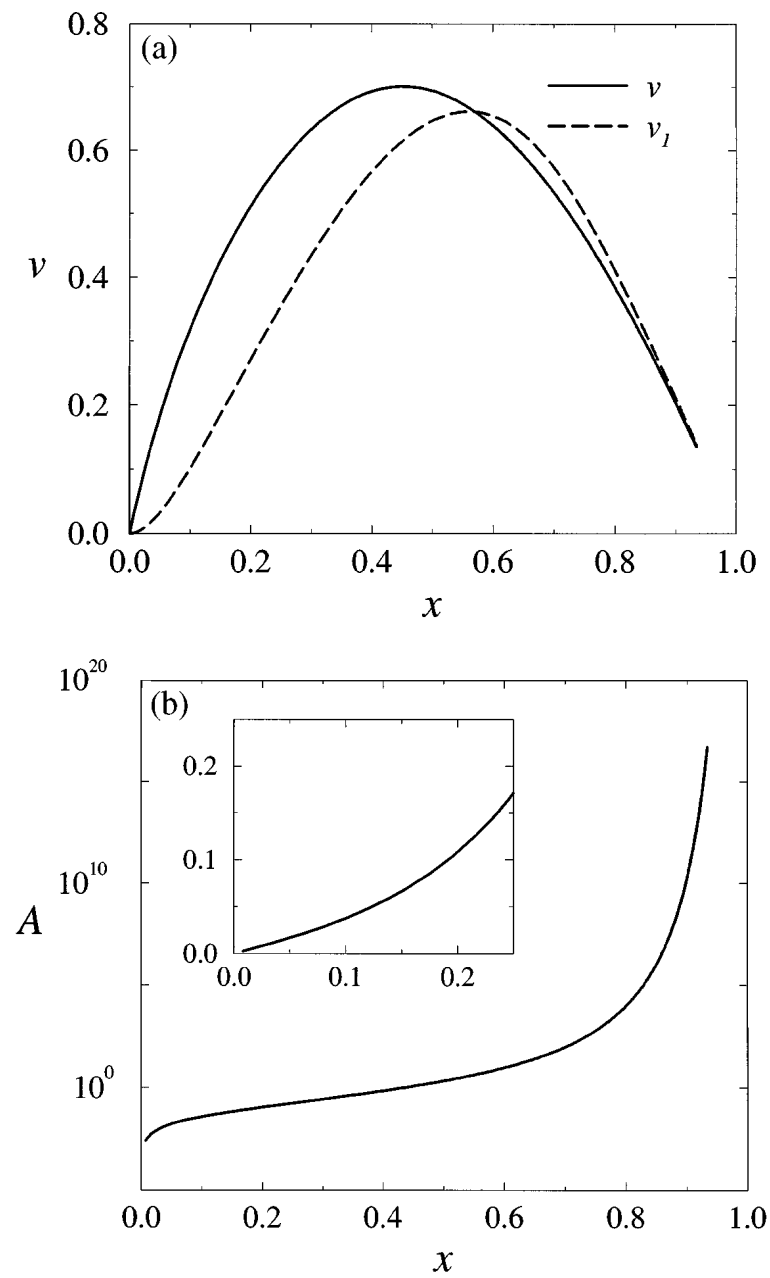

FIG. 2. Parameters of the effective model (17): (a) Velocities $v$ of the charge mode (16) and $v_{1}$ of the fermion $\chi_{0}$ (18) and (b) the parameter $A$ in the TBA (12) versus hole concentration $x$. The inset shows $A$ for small $x$. Note that $v_{2} \equiv \pi$ for all fillings. the integrable $S=1$ Takhtajan-Babujian and the $S=$ $1 / 2$ Heisenberg chain. For finite doping we find up to three massless modes determining the low temperature thermodynamics of the system. In the SU(2) invariant case $(H=0)$ these are a bosonic charge mode and a direct sum of a SU(2) 2 WZNW model and one Majorana fermion with different velocities in the spin sector. Further analysis of the TBA is necessary to gain more insight in the nature of the phase diagram of this and the related $S>1$ systems.

This work has been supported by the Deutsche Forschungsgemeinschaft under Grant No. Fr 737/2-2.

[1] K. Penc and H. Shiba, Phys. Rev. B 52, R175 (1995).

[2] E. Dagotto, J. Riera, A. Sandvik, and A. Moreo, Phys. Rev. Lett. 76, 1731 (1996).

[3] J. Riera, K. Hallberg, and E. Dagotto, Phys. Rev. Lett. 79, 713 (1997).

[4] J. F. DiTusa et al., Phys. Rev. Lett. 73, 1857 (1994).

[5] A. B. Zamolodchikov and V. A. Fateev, Sov. J. Nucl. Phys. 43, 657 (1986).

[6] V. J. Emery and S. Kivelson, Phys. Rev. B 46, 10812 (1992); P. Coleman, L. B. Ioffe, and A. M. Tsvelik, Phys. Rev. B 52, 6611 (1995); P. Azaria, C. Hooley, P. Lecheminant, C. Lhuilier, and A. M. Tsvelik (to be published).

[7] P. Coleman and A. J. Schofield, Phys. Rev. Lett. 75, 2184 (1995).

[8] M. Scheunert, W. Nahm, and V. Rittenberg, J. Math. Phys. 18, 155 (1977); M. Marcu, J. Math. Phys. 21, 1277 (1980).

[9] F. H. L. Eßler and V.E. Korepin, Phys. Rev. B 46, 9147 (1992); A. Foerster and M. Karowski, Nucl. Phys. B396, 611 (1993).

[10] V.E. Korepin, N.M. Bogoliubov, and A. G. Izergin, Quantum Inverse Scattering Method and Correlation Functions (Cambridge University Press, Cambridge, 1993).

[11] B. Sutherland, Phys. Rev. B 12, 3795 (1975).

[12] P. P. Kulish, J. Sov. Math. 35, 2648 (1986) [Z. Nauchn. Semin. LOMI 145, 140 (1985)].

[13] P.P. Kulish and E. K. Sklyanin, in Integrable Quantum Field Theories, Lecture Notes in Physics Vol. 151, edited by J. Hietarinta and C. Montonen (Springer-Verlag, Berlin, 1982), pp. 61-119.

[14] M. P. Pfannmüller and H. Frahm, Nucl. Phys. B479, 575 (1996).

[15] L. Takhtajan, Phys. Lett. A 87, 479 (1982).

[16] H. M. Babujian, Phys. Lett. A 90, 479 (1982); Nucl. Phys. B215, 317 (1983).

[17] M. Takahashi, Prog. Theor. Phys. 46, 401 (1971).

[18] H. J. de Vega and F. Woynarovich, J. Phys. A 25, 4499 (1992); S. R. Aladim and M. J. Martins, J. Phys. A 26, L529 (1993); H. J. de Vega, L. Mezincescu, and R. I. Nepomechie, Phys. Rev. B 49, 13223 (1994); P. Schlottmann, Phys. Rev. B 49, 9202 (1994).

[19] N. Andrei and A. Jerez, Phys. Rev. Lett. 74, 4507 (1995); A. Jerez, N. Andrei, and G. Zarand, cond-mat/9803137.

[20] A. M. Tsvelik, Phys. Rev. B 42, 10499 (1990); D. G. Shelton, A. A. Nersesyan, and A. M. Tsvelik, Phys. Rev. B 53, 8521 (1996). 